\title{
Le témoin face au défi de la vraisemblance. L'usage de l'ekphrasis chez une rescapée du génocide des Tutsi au Rwanda
}

Lucie Donckier de Donceel

\section{CpenEdition}

Journals

Édition électronique

URL : https://journals.openedition.org/rhetorique/1223

DOI : $10.4000 /$ rhetorique. 1223

ISSN : 2270-6909

Éditeur

UGA Éditions/Université Grenoble Alpes

Édition imprimée

ISBN : 978-2-37747-325-0

Référence électronique

Lucie Donckier de Donceel, «Le témoin face au défı de la vraisemblance. L'usage de l'ekphrasis chez une rescapée du génocide des Tutsi au Rwanda », Exercices de rhétorique [En ligne], 17 | 2021, mis en ligne le 25 novembre 2021, consulté le 29 novembre 2021. URL : http://journals.openedition.org/ rhetorique/1223; DOI : https://doi.org/10.4000/rhetorique.1223

Ce document a été généré automatiquement le 29 novembre 2021.

\section{c) (i) (8) (2)}

Les contenus de la revue Exercices de rhétorique sont mis à disposition selon les termes de la Licence Creative Commons Attribution - Pas d'Utilisation Commerciale - Partage dans les Mêmes Conditions 4.0 International. 


\section{Le témoin face au défi de la vraisemblance. L'usage de l'ekphrasis chez une rescapée du génocide des Tutsi au Rwanda}

Lucie Donckier de Donceel

«C'est parce que la catastrophe constitue un

destin

détestable dont nous devons dire que nous n'en voulons pas qu'il faut garder les yeux fixés sur

elle, sans jamais la perdre de vue. » Jean-Pierre Dupuy

\section{Introduction}

1 Cet article part du constat suivant: même si penser le génocide n'est pas nouveau, chaque massacre de cette nature reste un événement inédit dans sa singularité ${ }^{1}$. Or, comment faire pour raconter un événement qui est, par essence, unique, un événement qui, littéralement, ne ressemble à rien de connu ? Ce défi-ci, est d'un ordre particulier : il n'est ni juridique, ni historique, il est rhétorique. Le défi rhétorique que constitue l'expression d'un fait ineffable, ici génocidaire, a notamment été étudié par Marc Dominicy $^{2}$ et Emmanuelle Danblon ${ }^{3}$ qui, dans leurs travaux sur les témoignages des rescapés de la Shoah, ont souligné deux points. Ils remarquent le recours aux figures pour exprimer l'ineffable et ont qualifié ce défi, de défi pour la vraisemblance.

Dans cet article, nous nous proposons, à partir de ces deux considérations, d'analyser l'ekphrasis comme stratégie du témoin pour figurer l'invraisemblable. Plus précisément, 
nous soutiendrons que le recours à cette figure permet deux choses. D'une part, l'évocation mentale et sensorielle de ce que peut être un génocide. D'autre part, l'évocation d'une image mémorielle que l'on peut ensuite transmettre ${ }^{4}$. Enfin, la question de la transmission nous permettra de clôturer notre étude par une brève réflexion sur la fonction sociétale des témoins rescapés des grandes catastrophes humaines.

3 Cette étude sur la figure de l'ekphrasis se fera à partir d'un corpus précis - un extrait du témoignage de Félicité Lyamukuru, rescapée du génocide des Tutsi au Rwanda ${ }^{5}$ et en plusieurs temps. D'abord, il nous faudra poser quelques repères: des précisions historiques sont nécessaires pour situer le témoignage étudié. Ensuite, nous proposerons, à partir du témoignage, une réflexion sur le défi rhétorique induit par l'action de témoigner d'un événement unique de par son caractère singulier et inédit. Nous accorderons alors une attention particulière à la figure de l'ekpkrasis. Enfin, nous conclurons par une réflexion sur le rôle des témoins des grandes catastrophes humaines dans nos sociétés contemporaines.

\section{Cadre historique}

\section{Le génocide des Tutsi au Rwanda}

4 Pouvoir infirmer ou confirmer ces hypothèses nécessite d'entrer dans le grain fin de l'analyse du témoignage qui nous intéresse et pour ce faire, il est nécessaire de passer par un bref recadrage historique: quel est le contexte dans lequel s'inscrit ce témoignage, quel est le contexte du génocide des Tutsi au Rwanda. En 1994, le dernier génocide du $\mathrm{xx}^{\mathrm{e}}$ siècle, le génocide des Tutsi du Rwanda, se déroule dans l'indifférence quasi-totale de la communauté internationale. Pendant trois mois, d'avril à juillet, ce sont près d'un million de Tutsi qui sont massacrés par le pouvoir hutu en place, secondé par une partie de la population ${ }^{6}$.

5 Ce génocide, comme les précédents, se caractérise par une volonté d'extermination physique, culturelle et sociale de la population visée, par une essentialisation de cette population et par un discours génocidaire fortement imprégné par le langage médical et une idéologie hygiéniste : les Tutsi sont des serpents, des cafards, ils sont porteurs de maladies et de perversion, ce sont des nuisibles à éradiquer ${ }^{7}$. De plus, comme ce fut le cas avec les discours nazis, la parole génocidaire hutue propage l'idée d'une trahison nationale - les Tutsi, de supposée origine « hamite » auraient voulu prendre le contrôle non seulement du Rwanda mais aussi de l'ensemble de la région des Grands Lacs - et l'idée d'une « hiérarchisation » des races. Au Rwanda, comme en Europe auparavant, ce discours de haine se double d'une propagande efficace (à travers la radio RTML, le journal Kangura, dans les écoles ${ }^{8}$, etc.) et d'une préparation implacable. Tous les éléments sont réunis pour que l'ensemble des barrières morales et civilisationnelles qui, généralement, empêchent la barbarie de devenir le quotidien des sociétés, sautent ${ }^{9}$.

6 Pourtant, le génocide des Arméniens n'est pas celui des Juifs qui n'est pas celui des Tutsi. Les Tutsi et les Hutu parlent la même langue, partagent une histoire commune et une religion unique. La différence entre ces deux identités fut, en fait, largement construite et surtout figée par les colons, d'abord allemands et ensuite belges. Tandis qu'avant leur arrivée " hutu » et «tutsi » faisaient référence à des catégories socioéconomiques souples, au moment de la décolonisation, ces identités étaient devenues 
immuables. Dans un esprit de « catégorisation » des races, le colon chercha à distinguer physiquement, socialement et historiquement le Hutu du Tutsi. Désormais on naissait l'un ou l'autre et cela était inscrit sur la carte d'identité de chacun. D'une appellation socio-économique, les termes de «tutsi » et " hutu » étaient devenus des catégories ethniques. La fixation de ces identités et le discours de la Belgique dans les années entourant l'indépendance du Rwanda ont également fortement nourri le sentiment de haine des Hutu envers les Tutsi qui, par la suite, engendra des massacres réguliers des Tutsi par les $\mathrm{Hutu}^{10}$. Ces massacres furent pendant longtemps impunis et préparèrent le terrain à l'extermination systématique de la population tutsie en $1994^{11}$.

7 Outre un contexte historique particulier, le génocide des Tutsi au Rwanda, génocide « au village » comme le nomme Hélène Dumas ${ }^{12}$ se caractérise également par une très forte participation des acteurs locaux aux tueries: la mobilisation de tout un savoir populaire au service de la mort des proches. Du gouvernement national au bourgmestre, en passant par le voisin, c'est la population toute entière qui prend part aux massacres et la machine exterminatrice est d'autant plus efficace au cœur des collines que le Tutsi voisin et collègue y est transformé en ennemi et sa descendance impitoyablement exterminée. Il semblerait que cette proximité entre bourreaux et victimes, loin de générer un sentiment d'empathie, au contraire ait accru la souffrance pour les victimes et la cruauté pour les bourreaux.

\section{Le témoignage de Félicité Lyamukuru}

8 C'est dans ce contexte de massacres entre voisins que se situe le témoignage de Félicité Lyamukuru. Rescapée du génocide des Tutsi, sa famille fut tuée dans la soirée du 7 avril 1994, au Petit Séminaire de Nyundo, en bordure du lac Kivu. D'une fratrie de sept frères et sœurs, seule Félicité et l'un de ses petits frères survécurent.

9 Son témoignage nous est parvenu sous la forme d'un récit écrit à quatre mains, en français, par elle et Nathalie Caprioli ${ }^{13}$ entre 2014 et 2016, et finalement publié en 2018 aux éditions du Cerisier. Bien que sur un ton relativement brut et principalement à la première personne, ce récit est émaillé de dialogues : entre Félicité et Nathalie, entre Félicité et d'autres personnes - présentes ou absentes -, entre le passé et le présent mais aussi entre le témoignage de Félicité et les éléments historiques et didactiques du récit. À ce stade-ci, il nous semble également important de souligner que certains passages du récit, (notamment à l'évocation de la mort de sa mère par certaines nounous de l'orphelinat $\left.{ }^{14}\right)$, ont fait l'objet d'une autocensure de la part de Félicité, autocensure qui s'explique sans doute par l'idée de convenance mais aussi de cruauté du réel et de peur de ne pas être crue ${ }^{15}$. Cependant, sur les conseils de Nathalie Caprioli, ces souvenirs ont quand même pris corps à l'écrit et se retrouvent dans le récit qui nous intéresse. Ces quelques précisions faites, nous allons maintenant nous concentrer sur le récit, par Félicité, du massacre de sa famille.

La nuit du 6 au 7 avril 1994, l'avion présidentiel fut abattu et les présidents rwandais et burundais, respectivement J. Habyarimana et C. Ntaryamira, perdirent la vie. Dans un contexte de tensions extrêmes entre forces gouvernementales à majorité hutue - les Forces Armées Rwandaises - et exilés rwandais principalement tutsis - le Front Patriotique Rwandais -, cet attentat, premièrement attribués au $\mathrm{FPR}^{16}$, fut considéré comme la provocation de trop, comme le prétexte et l'élément déclencheur du génocide des Tutsi. 
11 Le lendemain de l'attentat, le souvenir des précédents massacres de Tutsi ${ }^{17}$ en tête, Félicité et sa famille se réfugient au Petit Séminaire; les lieux de culte ayant toujours été, jusque-là, des lieux de protections et d'asile ${ }^{18}$. Pourtant, dans la soirée du 7 avril, une milice génocidaire entre dans le Petit Séminaire et massacre une quarantaine de personnes, toutes cachées dans une chapelle, dissimulée par une porte en trompe-l'œil. De cette cachette, 6 personnes survécurent, dont Félicité, sortie pour aller chercher un pull et qui, effrayée par le bruit, se réfugia dans un faux-plafond.

12 L'extrait du témoignage de Félicité ${ }^{19}$ fait écho à ce massacre, elle nous le raconte sans explicitement le détailler, elle nous conte les souvenirs qu'elle garde des moments précédant l'attaque, ainsi que sa réaction à la découverte de la cachette profanée par les tueurs et devenue lieu d'extermination.

[...]

[1] La chapelle faisait à peine trente mètres carrés. On aurait dit une classe meublée

[2] d'une table et d'un autel qui ressemblait plus à un bureau d'instituteur. Presque

[3] une quarantaine, nous étions les uns sur les autres, à même le sol, essayant

[4] d'allonger les jambes tant bien que mal. La femme de mon oncle Alfred installa

[5] les enfants par groupe d'âge et s'occupa des plus petits. Avec mes copines et

[6] Alphonsine, nous commençâmes à jouer aux cartes. Nous ne parlions pas et je

[7] veillais à garder les yeux baissés pour ne pas lire la peur dans nos regards

[8] respectifs. Qu'allait-il se passer ? Personne n'en avait la moindre idée.

[9] Ma mère entama une tournée de lait auprès des enfants. J'étais plongée dans la

[10] partie quand elle arriva à mon niveau et articula « lève-toi! »

[11] - Quoi ? Je joue !

[12] - Je veux te servir du lait, mais avant tu vas dans le bureau de papa, tu prends un

[13] pull. Sinon tu vas piquer une crise d'asthme et on ne s'en sortira pas.

[14] - Je t'en supplie, laisse-moi terminer ce jeu. On est au bout !

[15] - Non. Tu vas chercher ton pull.

[16] Ma sœur déposa son jeu et mélangea les cartes : partie terminée. Je me levai

[17] d'autant plus énervée que j'étais sur le point de gagner.

[18] - Je bois mon lait, puis j'y vais.

[19] C'est que je commençais à sentir la faim.

[20] - Non. Tu vois ce lait ? Tu le boiras quand tu rentreras avec ton pull.

[21] Elle était inflexible ! Malgré mon regard fâché, ma mère posa un baiser sur mon

[22] front et le verre sur le rebord de la table. Avant de quitter la pièce, j'ai eu le

[23] temps d'observer que chacun avait fini par se calmer. Dans cette chapelle,

[24] personne ne nous trouverait parce que personne ne connaissait la porte dérobée.

[...]

[25] Mes genoux se dérobèrent. Je n'ai pas pu crier. Je ne comprenais rien. J'aurais

[26] aimé fermer les yeux, le temps que tout s'arrange. Dans la pièce, les néons me

[27] brûlaient les yeux. Je vis d'abord ma mère contre la porte. Elle ne respirait plus. À

[28] son côté, ma petite sœur Jeanne d'Arc et ma cousine Alice : mortes. Ma grande

[29] sœur Alphonsine n'était pas loin : morte. Du couloir, j'entendais les prêtres et

[30] militaires trépigner : « Mais enfin, qu'est ce qu'elle fabrique? »

[31] Mon père entra à son tour. Il resta lui aussi sans voix devant ces corps

[32] démantibulés. Le réel explosa à mes yeux. C'était la première fois que je voyais

[33] la mort de si près : d'un coup, des dizaines de corps.

[34] Les lumières étaient si fortes que les détails m'apparaissaient surdimensionnés.

[35] Tonton Alfred gisait sous la table, sur le coin de laquelle ma mère avait 
[36] déposé le verre de lait resté intact. Alfred, le seul papa dans cette chapelle

[37] que tous avaient crue inviolable, agonisait, bras et jambes coupés.

$[\ldots]$

\section{Témoigner d'un événement singulier : un défi pour la vraisemblance}

\section{Figurer un « possible invraisemblable »} défi bien particulier, celui de transmettre le récit d'un événement qui est toujours singulier, qui ne ressemble à rien de connu pour les auditeurs. En termes rhétoriques, les témoins rescapés des grandes catastrophes humaines, dans notre cas, du génocide des Tutsi, sont confrontés à cette difficulté : comment faire pour exprimer un possible invraisemblable, c'est-à-dire un événement qui a eu lieu mais qui n'évoque aucune image dans l'esprit des auditeurs ${ }^{20}$.

Comprendre ce que veut dire invraisemblable implique de passer par son corollaire opposé, le vraisemblable. Bien qu'objet de nombreuses discussions parmi les spécialistes $^{21}$, nous nous référerons ici à la définition antique de la vraisemblance telle qu'exposée dans la Rhétorique à Alexandre ${ }^{22}$ : «Le vraisemblable est ce dont quand on le dit, les auditeurs ont des exemples en tête" (Rh. Al., 7, 1-4). Définition antique du vraisemblable qui correspond presque mot pour mot à la définition contemporaine qu'en propose Francis Goyet : « le vraisemblable n'est pas le vrai, mais ce qui ressemble à l'idée - au type - que le public s'en fait ${ }^{23}$ ». Le génocide des Tutsi au Rwanda serait, comme beaucoup l'ont affirmé à propos de la Shoah, un événement invraisemblable au premier sens du terme, littéralement, un événement qui ne ressemble à rien. Un événement qui, lorsque raconté, n'évoque aucun "type ", aucune idée, aucune image, dans l'esprit des auditeurs. Car, même si le génocide des Tutsi succède au génocide des Arméniens et à celui des Juifs, il ne faut pas s'y méprendre, les massacres génocidaires sont toujours uniques et n'évoquent jamais une idée générale du crime commis. Les rescapés du génocide des Tutsi se trouvent donc devant le même obstacle que les rescapés de la Shoah : comment raconter un possible invraisemblable, comment mettre en mots un vécu dont la dureté et la cruauté n'évoquent rien dans l'esprit des auditeurs? Il semblerait que la tâche ardue d'exprimer un possible invraisemblable de le transformer en possible vraisemblable - soit réalisable si l'orateur recours aux figures rhétoriques pour transmettre son vécu ${ }^{24}$.

Le recours aux figures, parmi lesquelles on peut citer notamment la parabole, l'énigme, l'amplification et l'ekphrasis, permettrait d'exercer un regard lucide sur un événement ${ }^{25}$. À travers des effets de zoom-avant sur certains éléments et de zoomarrière sur le tableau d'ensemble, le témoin pourrait dépeindre un tableau d'ensemble compréhensible, vraisemblable, pour les auditeurs. Cet effet des figures est ce que Francis Goyet traduit par les notions d'omnia et de totum: le totum c'est le concept d'ensemble, les omnia ce sont les détails. Et bien qu'accéder au totum ne soit pas une chose simple, il existerait une consigne - que Goyet emprunte à Quintilien - pour mettre en œuvre ces deux concepts : développer des détails concrets permet un passage du visible à l'invisible, des omnia au totum «puisque dans l'énumération, nous allons progressivement, linéairement, du concret au concept, de la foule des détails atroces à 
une vue d'ensemble de la situation; tout comme le médecin remonte du visible, le tableau des symptômes, à l'invisible quand il identifie et nomme la maladie ${ }^{26} »$. Accéder à une vision d'ensemble juste, la comprendre, demande l'interprétation des détails qui la constituent. Autrement dit, si nous n'effectuons pas ce détour par les détails, nous restons en surface de l'événement que l'on nous expose, nous ne le comprenons pas ${ }^{27}$. Or, s'assurer que l'auditoire ait accès au totum est une tâche d'autant plus ardue quand le message que l'orateur cherche à transmettre relève du possible invraisemblable. Pourtant, il nous semble que Félicité effectue justement ce travail d'aller-retour afin de nous permettre, à travers certains omnia, certains éléments, cet accès au totum. Ces éléments sont nombreux : certains participent d'un effet général de compréhension du discours ${ }^{28}$ et d'autres participent selon nous, plus clairement du passage des omnia vers le totum. C'est, nous le proposons, le rôle que joue la figure de l'ekphrasis dans ce témoignage.

\section{Le recours à la figure de l'ekphrasis}

La figure de l'ekphrasis dans le témoignage qui nous occupe se matérialise dans le « verre de lait » que Félicité mentionne à plusieurs reprises ([9]; [12]; [18]; [20]; [22]; [36]). Ce détail au sein de son récit est selon nous, un des omnia participant à la compréhension du totum, sous la forme d'une ekphrasis, figure qui permettrait dans le témoignage qui nous occupe la représentation d'un possible invraisemblable.

Comme le rappelle Ruth $\mathrm{Webb}^{29}$, la signification du terme ekphrasis a toujours fait l'objet de multiples interprétations. Si, actuellement, critiques et historiens de l'art discutent pour savoir ce que cette notion recouvre en termes d'images et de mots, des débats similaires animaient déjà les Anciens en leurs temps. Néanmoins, pour le propos de notre article, nous adopterons, dans un premier temps, une définition large de cette figure, définition que nous nuancerons ensuite en termes d'effets sur l'auditoire. Nous aborderons d'abord l'idée que la figure étudiée participe du partage des émotions entre orateur et auditeurs. Ensuite, s'appuyant sur l'analyse que propose Ruth Webb d'un groupe d'ekphraseis de l'Antiquité tardive de sculptures répertoriées dans l'œuvre de Libanios, nous explorerons la piste d'un recours à l'ekphrasis pour évoquer un contexte narratif plus large ${ }^{30}$.

\subsection{Partager l'émotion de l'orateur}

Généralement, l'ekphrasis dans sa conception rhétorique, telle que définie dans différents progymnasmata ${ }^{31}$, fait allusion à un discours détaillée et animé, un discours vivant qui mettrait devant les yeux de l'auditoire la chose racontée. À cette première appréhension de la figure s'ajoutent deux traits fondamentaux : l'ekphrasis ne concerne pas uniquement le domaine du statique - elle peut concerner une personne, un endroit, un moment ou un événement - et surtout, elle est définie en termes d'impact sur l'auditoire : la description vivante, à travers la qualité d'enargeia doit participer de la compréhension de la chose décrite. L'auditeur doit, à travers l'ekphrasis, voir la chose qu'on lui décrit. En effet, selon Quintilien à qui Ruth $\mathrm{Webb}^{32}$ fait référence, un orateur vertueux est un orateur capable de produire un discours décrivant une chose de façon si vivace que celle-ci se dessine devant les yeux de l'auditoire. Le mécanisme discursif de la description détaillée serait l'ekphrasis et l'effet d'évidence ${ }^{33}$ produit par le discours, ce qu'il nomme l'enargeia. Plus spécifiquement, l'auteur ${ }^{34}$ précise que l'enargeia 
est produite quand un orateur recourt à sa propre imagination pour décrire une chose de façon telle que l'auditoire a l'impression d'avoir lui-même accès à la chose décrite (Quintilien, Institutio oratoria, 6.2.29-32). Autrement dit, pour l'auteur antique, les discours ayant cette qualité d'enargeia sont des discours qui, à travers la description détaillée, mettent en mots une image présente dans l'esprit de l'orateur de façon telle que cette même image apparaisse dans l'esprit de l'auditoire. Le recours à l'ekphrasis contraste donc avec un simple exposé des faits en ce qu'elle fait voir au public la chose qu'on lui décrit ${ }^{35}$. L'auteur illustre notamment ses dires à l'aide de l'exemple courant dans l'Antiquité du sac d'une ville : c'est une chose de dire qu'une ville est tombée mais c'en est une autre d'en montrer les détails au public de façon telle qu'il sente et voie, qu'il comprenne ce qu'est le sac d'une ville. Et Quintilien insiste, afin de provoquer cet effet de présence, un orateur doit s'assurer que la chose qu'il décrit à l'auditoire fasse écho à des connaissances et représentations préalables disponibles dans l'esprit de l'auditoire (Quintilien, 6.2.30). En effet, comme le souligne Ruth Webb, recourir à l'ekphrasis est une opération délicate: l'impact de cette figure, la qualité d'enargeia découle du choix judicieux de détails qui correspondent à des connaissances et attentes préalables du public, faisant appel aux images mentales stockées dans la mémoire de tout un chacun ${ }^{36}$.

Or, que faire quand l'image que l'on veut mettre devant les yeux de l'auditoire ne peut être rattachée à une image mentale déjà disponible? Que faire quand la situation que l'on décrit est proprement invraisemblable, ce qui est souvent le cas dans les témoignages des rescapés des grandes catastrophes humaines? La solution qui nous semble être adoptée dans le témoignage qui nous occupe ici est, comme soutenu auparavant, le recours aux figures. Plus précisément, Félicité aurait recours à l'ekphrasis non pas pour évoquer l'image mentale du génocide à proprement parler, mais bien le sentiment d'invraisemblance qui émane du génocide.

Cette hypothèse nous semble renforcée par le fait que, comme le souligne Nikolaos ${ }^{37}$, le recours à l'ekphrasis a pour but de placer la chose décrite devant les yeux de l'auditoire, mais aussi, de lui faire ressentir ce que l'orateur a éprouvé face à la chose décrite. Comme le souligne Nikolaos à propos du sac de la ville de Phokios par Philippe de Macédoine, "voir le tout ", c'est aussi partager le sentiment d'indignation qui frappait les citoyens face à cette scène ${ }^{38}$. En effet, comme le soulignaient déjà les Anciens ${ }^{39}$, les émotions participent de la persuasion et donc, comprendre le tout implique de voir la chose décrite dans toutes ses dimensions, sensorielles et mentales ${ }^{40}$. Nous pensons que la présence répétée du verre de lait dans la chapelle du Petit Séminaire de Nyundo participe de cette logique. L'évocation discursive de sa présence nous implique aussi au niveau émotionnel : nous sommes confrontés à un sentiment d'incompréhension et de désarroi. Textuellement, nous nous demandons : comment ce verre de lait a-t-il pu rester intact malgré le massacre qui faisait rage tout autour? Le temps du témoignage, nous partageons la stupeur et l'incompréhension de Félicité face à la scène de carnage qu'elle découvrait. Enfin, comme le souligne Marc Dominicy ${ }^{41}$, nous sommes peut-être confrontés ici à un remodelage poétique de la vérité sémantique: il est très peu vraisemblable que ce verre de lait soit resté intact et immaculé malgré le massacre et pourtant, cela importe peu, au contraire, cela renforce l'idée qu'un génocide soit brutal et incompréhensible pour ceux qui en sont les victimes.

21 L'hypothèse selon laquelle le recours à l'ekphrasis participe d'une volonté de l'orateur de nous faire partager son sentiment d'incompréhension et de désarroi face à la scène 
dont elle est témoin, et donc participe à l'évocation de l'invraisemblable, est renforcée par la réflexion qu'Emmanuelle Danblon propose sur l'effet de décalage observé dans les témoignages des rescapés de la Shoah ${ }^{42}$. En effet, et cette idée est également présentée par Marc Dominicy, il semblerait que les témoins des grandes catastrophes humaines puissent avoir recours à différents ressorts lexicaux et figuratifs pour provoquer un effet de choc dans l'auditoire; effet de choc qui évoquerait la brutalité de l'événement. Ce contraste, ici entre le blanc du verre de lait et le rouge sang des massacres ${ }^{43}$, mais aussi entre l'évocation du monde de l'enfance, en mouvement, et la raideur et froideur du monde de la mort, « permettrait au lecteur de penser le génocide à partir du saut brutal qui mène de la vie réelle à l'irréalité de la mort ${ }^{44}$ ». Et en effet, à plusieurs reprises Félicité fait allusion à cet univers et le convoque : référence à la salle au monde scolaire, «La chapelle faisait à peine trente mètres carrés. On aurait dit une classe meublée d'une table et d'un autel qui ressemblait plus à un bureau d'instituteur » [1-2]; allusion au jeu de cartes et à la compétition enfantine, «Avec mes copines et Alphonsine, nous commençâmes à jouer aux cartes " [5-6], "Je t'en supplie, laisse moi terminer ce jeu " [14], «Je me levai d'autant plus énervée que j'étais sur le point de gagner » [17]; évocation de réalités propres aux relations parents-enfants [ $m a$ mère entama une tournée de lait auprès des enfants " [9], « ma mère posa un baiser sur mon front » [21], «Alfred, le seul papa de cette chapelle » [36]. Ensuite, le monde de l'enfance laisse place à un registre lexical direct, cru et froid et à une amplification qui décrivent la mort et la violence du génocide : "elle ne respirait plus» [27]; «mortes, morte» [28-29]; "ces corps démantibulés » [31]; "bras et jambes coupés » [37]. La présence du verre de lait tout comme le choix du lexique participent selon nous, de la construction du sentiment d'incompréhension, ils contribuent à dessiner une situation possible mais invraisemblable.

\section{2. Évoquer un contexte narratif plus large}

Nous le disions, nous soutenons qu'à travers la présence de ce verre de lait, c'est toute l'invraisemblance du génocide que le témoin veut partager avec nous. Pourtant, il nous faut aller un pas plus loin. Arriver à une telle conclusion suppose de notre part un travail d'inférence : du verre de lait, nous passons au génocide. Ce travail d'inférence, d'interprétation est, comme l'expose Nikolaos toujours suite à l'évocation du sac de la ville de Phokios par Philippe de Macédoine, l'un des traits caractéristiques du fonctionnement de l'ekphrasis. C'est ce que Ruth Webb souligne dans le passage suivant ${ }^{45}$ :

Ce qui est remarquable dans ce passage, et d'une grande importance pour le fonctionnement de l'ekphrasis en général, c'est que l'action face à laquelle nous sommes censés nous indigner [la violence de Philippe de Macédoine] n'est pas décrite directement mais à partir des traces qu'elle a laissées. Or, pour que le recours à l'ekphrasis soit efficace, il faut que le public fournisse lui-même cette information. Les détails décrits sont donc principalement importants en tant que signes d'autre chose, et nous sommes laissés libres d'en déduire les actions qui les ont précédés.

Ce mécanisme d'interprétation est donc supposé induit par le recours même à la figure de l'ekphrasis. Or, il semblerait que dans certains cas, l'interprétation de l'ekphrasis soit induite par l'orateur dans la description qu'il propose. C'est ce que Ruth Webb observe à propos des ekphraseis tardives de sculptures, attribuées à Libanios et sans doute 
produites dans un cadre scolaire. Elles ne laissent pas ou peu de place à l'interprétation.

Par exemple (Libanios, Opera VIII 5064) :

Dans le cas de la femme troyenne, le sein découvert montrerait que ses malheurs ont partiellement effacé son sentiment de honte (aidōs) et le narrateur spécule qu'elle n'est pas consciente de sa nudité partielle - une remarque qui la dépeint comme l'objet involontaire du regard masculin.

Ce qui nous intéresse ici est l'hypothèse que Ruth $\mathrm{Webb}^{47}$ construit à partir de ces groupes d'ekphraseis dotées de «sous-textes ». Elle suggère que de telles ekphraseis, audelà de la pure description détaillée, participent de l'évocation d'un contexte narratif plus important. Plus précisément, ces ekphraseis ne constitueraient ni des grilles de lecture de l'art de l'époque, ni des grilles de lecture des réalités de l'époque, mais seraient plutôt le fruit d'une volonté d'instruire l'auditoire, le fruit d'une volonté de transmission d'un savoir attaché à un contexte et une époque particulière. Son hypothèse, bien que non attestée telle quelle dans les sources antiques (mais appuyée par l'idée selon laquelle "l'ekphrasis doit provoquer des images marquantes afin qu'elles soient mémorables ${ }^{48} »$ ) est que ces ekphraseis permettraient la construction et la transmission d'un savoir en agissant comme des « images mémorielles». Ces « images mémorielles » produites à travers le recours à l'ekphrasis auraient donc pour but la transmission et préservation de connaissances.

Ceci dit, nous pensons pouvoir déceler une même intention dans le témoignage de Félicité qui, face à la scène de massacre, déclare «Je ne comprenais rien ${ }^{49}$ ". Tandis que ce marqueur pourrait simplement indiquer un échec de la vraisemblance (Félicité voit les omnia, les détails des corps massacrés, mais elle n'a pas accès au totum, à une compréhension générale du tableau qui s'offre à elle), nous pensons qu'il s'agit surtout d'un indicateur du sentiment ressenti par le témoin face à la scène qu'elle découvre, et que cet indicateur serait analogue à la lecture que nous propose Libanios de la statue de la femme troyenne. Félicité nous explique ce que nous devrions ressentir face à un tel massacre, et par extrapolation, face au génocide des Tutsi au Rwanda. Nous postulons donc que l'image du verre de lait resté immaculé dans la tourmente du massacre évoque, d'une part, la construction d'une représentation de l'invraisemblable et, d'autre part, puisse être perçue comme une «image mémorielle " du génocide, une image permettant de fixer la brutalité de tels événements dans l'esprit des auditeurs.

\section{Quelle fonction pour les témoins rescapés des grandes catastrophes humaines?}

Repartons de l'hypothèse selon laquelle le rôle de l'ekphrasis serait d'inscrire certains événements au sein de la mémoire collective. Inscrire un récit du génocide dans la mémoire de tous participerait de la construction d'une mémoire culturelle au sens où Ian Assmann ${ }^{50}$ la définit. Par opposition avec la mémoire communicationnelle - celle du souvenir vécu qui s'incarne dans les témoins directs -, la mémoire culturelle est celle du souvenir façonné et étayé par mnémotechnie culturelle. Cette terminologie fait référence à ce que Ian et Aleida Assmann - à la suite de Jurij Lotman - qualifient de situation de communication distendue, ce qui implique des possibilités d'enregistrement intermédiaires externes. Concrètement, le système de communication doit développer des modes de préservation des messages et des informations et alors seulement se constitue une mémoire qui déborde plus ou moins largement l'horizon du sens 
transmis à chaque époque, et dépasse la communication, la mémoire communicationnelle. Poussant la réflexion un pas plus loin, le penseur allemand précise $^{51}$ :

Une vérité, pour se fixer dans la mémoire d'un groupe, doit se présenter sous la forme concrète d'un événement, d'une figure personnelle, d'un lieu. Mais à l'inverse, pour perdurer dans la mémoire d'un groupe, un événement doit acquérir la charge de sens d'une vérité significative. Tout personnage et tout fait historique, dès qu'il pénètre dans cette mémoire s'y transpose en un enseignement, en une notion, en un symbole ; il reçoit un sens ; il devient un élément d'idées de la société.

Pour notre propos, nous faisons l'hypothèse d'un recours à l'expression poétique, une figure rhétorique, pour transmettre la mémoire des événements et opérer ce passage de la mémoire communicationnelle à la mémoire culturelle. En effet, c'est le recours à l'expression poétique, ici sous la forme de l'ekphrasis, qui permettrait de rapprocher les récits des rescapés des grandes catastrophes humaines d'une "mémoire symbole" comme la qualifie Assmann. Allant un pas plus loin, la notion de "mémoire symbole » permettrait de rapprocher les témoins des grandes catastrophes humaines de la catégorie antique, définie par Aristote, des témoins anciens. À l'inverse des témoins actuels qui sont ceux qui ont vu l'événement, ceux que l'on qualifierait dans le jargon juridique contemporain de témoins oculaires ${ }^{52}$, les témoins anciens - parmi lesquels on trouve les poètes - sont des figures de références pour une communauté donnée, ceux que l'on invoque sans que leur parole ne soit mise en doute, ceux qui font partie du patrimoine culturel d'une société donnée (Aristote, Rhétorique I, 15, 1375b28-3553). La proposition de classer les témoins rescapés des grandes catastrophes humaines parmi les témoins anciens semble être confirmée par la définition que propose Renaud Dulong $^{54} \mathrm{du}$ témoignage historique. Il s'agit d'un témoin qui, bien qu'il s'adresse à ses contemporains, parle également aux générations futures. Le contenu du témoignage n'est pas, en ce sens, dirigé vers une institution spécifique mais aurait une portée universelle: il s'adresse à qui voudra bien le recevoir. De plus, l'auteur soutient également que ce qui importe dans le témoignage historique est que "la "vérité" du témoignage sur les catastrophes renvoie moins au détail des descriptions qu'à la mémoire globale de ce qui s'est passé et n'aurait jamais dû arriver. " Les deux auteurs mobilisés évoquent ici, chacun à leur manière, les fonctions de l'ekphrasis étudiées : la création d'une image nous permettant de nous figurer l'invraisemblance d'un génocide et la création d'une image mémorielle qui nous permet d'inscrire cet événement dans notre patrimoine culturel et historique, notre mémoire culturelle.

\section{Conclusion}

Nous le soulignions en début d'article, bien que depuis un demi-siècle environ, juristes, philosophes, historiens, psychologues, dédient de nombreux travaux à la question du génocide, un massacre de cet ordre, que l'on considère celui des Arméniens, des Juifs ou des Tutsi, est toujours un événement singulier. Et face à la singularité, un double défi est constant : celui de l'expression et de la transmission. Comment faire pour figurer un événement à la fois unique et invraisemblable? Cet enjeu se présente à chaque génération de témoins rescapés des grandes catastrophes. Nous avons cherché à montrer que la nature de ce défi est proprement rhétorique, tant pour le défi de l'expression que pour celui de la transmission. 

rescapés des grandes catastrophes humaines d'affronter le défi de l'expression de l'ineffable, la figuration de l'invraisemblable. Comme le soutiennent à la fois Marc Dominicy et Emmanuelle Danblon, faire un usage discursif des figures rhétoriques permettrait l'accès au totum, la figuration d'un tableau d'ensemble, par le détour par les omnia, les différents éléments composants la scène. Parmi les figures à disposition de l'orateur l'ekphrasis sera privilégiée. Elle répond en effet à deux fonctions rhétoriques essentielles: participer à la représentation de l'invraisemblable et façonner des « images mémorielle».

Au final, mobiliser la figure de l'ekphrasis dans cette double optique nous aura permis de contribuer à la réflexion sur le rôle des témoins rescapés des grandes catastrophes humaines pour nos sociétés. Leur rôle, que nous proposons de rapprocher de celui des témoins anciens tels que définis par Aristote, se décline ainsi en deux temps. D'abord, exprimer l'ineffable, ensuite, diffuser un message à vocation universelle.

Le message de Félicité, comme celui des autres témoins rescapés des grandes catastrophes humaines, touche profondément à notre humanité. Comme le note Renaud Dulong, ils témoignent d'histoires qui ne sont pas ordinaires, ils témoignent de catastrophes perpétrées par des hommes contre des hommes, ils témoignent de faits qui sont à la limite de ce que peut supporter un être humain. Ils exigent donc le recours à des stratégies rhétoriques elles aussi exceptionnelles.

\section{NOTES}

1. Pour nourrir la réflexion sur cette notion de singularité, voir également la réflexion que propose Renaud Dulong quant à la figure du témoin historique (R. Dulong, "Qu'est-ce qu'un témoin historique?» dans A. Prstojevic dir., Vox Poetica, sans date [en ligne : http://www.voxpoetica.org/t/articles/dulong.html]) : «Dans cette classe de témoins, à la différence des deux autres pour lesquelles des schémas simples encadrent l'activité de témoigner, on a affaire à une collection d'individualités qui ne sauraient se laisser enfermer dans des traits génériques; ne serait-ce que parce que les situations qu'il s'agit de décrire ou les histoires qu'il faut raconter ne sont pas ordinaires. Car on ne parle de témoins historiques que pour des catastrophes perpétrées par des hommes contre des hommes, il ne s'agit pas d'accidents ponctuels qui peuvent arriver à tout un chacun, mais de l'Histoire venant s'emparer de la trajectoire d'un homme et éventuellement lui inspirer la possibilité de devenir chroniqueur de faits à la limite de ce que peut supporter un être humain. »

2. M. Dominicy, Poétique de l'évocation, Paris, Classiques Garnier, 2011.

3. E. Danblon, Mandorla de Paul Celan. Ou l'épreuve de la prophétie, Lormont, Le bord de l'eau, 2017.

4. Sur ce point, nous nous référons à la proposition faite par Ruth Webb dans son article «The Model Ekphraseis of Nikolaos the Sophist as Memory Images ", dans M. Grünbart (éd.), Rhetorical Culture in Late Antiquity and the Middle Ages, Berlin, De Gruyter, 2007, p. 463-476.

5. F. Lyamukuru \& N. Caprioli, Nathalie, L'ouragan a frappé Nyundo, Mons, Éditions du Cerisier, 2018. 
6. A. Des Forges, Aucun témoin ne doit survivre. Le génocide au Rwanda. Rapport pour Human Rights Watch et la Fédération internationale des Ligues des droits de l'Homme, Paris, Éditions Karthala, 1999.

7. On pensera de la même façon aux Juifs que l'on qualifiait de "vermine ", ou à la terrible remarque négationniste de Faurisson qui affirmait qu'au «bagne d'Auschwitz on a gazé ... des poux ». Interview de R. Faurisson pour l'émission 19/20 2eme, 30 avril 1987 : disponible sur les archives de l'INA: https://www.ina.fr/video/CAC01036119/interview-faurisson-campsconcentration-video.html (consulté le 16 novembre 2020).

8. À ce propos, le témoignage de Joan Murungi, membre du Rwanda Education board, est édifiant. Elle y rappelle que la préparation du génocide s'ancre notamment dans les écoles où, par exemple, un professeur de mathématique pouvait demander à ses élèves : « Si vous avez 10 Tutsi et que vous en tuez 5 , combien en reste-t-il ?» Témoignage présenté dans le cadre du film Nés pendant les jours sombres, documentaire réalisé par André Bossuroy pour Ibuka Mémoire \& Justice (2020) : https://www.ibuka.app/voir-le-film/.

9. Voir à ce propos les écrits de Florent Piton pour une chronologie des faits : F. Piton, Le génocide des Tutsi du Rwanda, Paris, La Découverte, 2018 ; et ceux de Vincent Duclert pour une réflexion sur la portée symbolique de tels événements: V. Duclert, "Les discours génocidaires ", Rhétorique. Origines et évolution, dans Textes et documents pour la classe $n^{\circ} 1125$, Paris, Éditions Canopé, 2019, p. 56-59.

10. Un premier pogrom cause la mort de dix mille Tutsi en 1963. Ces pogroms se répèteront en 1973, 1990, 1991, 1992 et 1993 (F. Lyamukuru \& N. Caprioli, L'ouragan a frappé Nyuido, op. cit., p. 286).

11. F. Piton, Le génocide des Tutsi du Rwanda, op. cit.

12. H. Dumas, Le génocide au village. Le massacre des Tutsi au Rwanda, Paris, Seuil, 2014.

13. Nathalie Caprioli, journaliste et co-auteur du témoignage qui nous occupe, est également depuis 2004 responsable de rédaction pour l'Agenda interculturel publié par le Centre bruxellois d'action interculturelle. (www.cbai.be).

14. À la page 174 de son livre, Félicité évoque l'attitude menaçante et méprisante des autres pensionnaires de l'orphelinat où elle et quelques autres enfants tutsis avaient trouvé refuge. Lors d'une de ces séquences, un groupe de nounous évoque la mort d'un groupe de femmes tutsies parmi lesquelles se trouvait la mère de Félicité. Félicité nous expliquera ultérieurement qu'elle ne voulait pas, au départ, que ce passage se retrouve dans ce récit car trop intime. En termes rhétoriques, nous pourrions rattacher cela au lieu de la convenance. Pour une réflexion sur la censure et la convenance, voir notamment J.-B. Amadieu, «Échappe-t-on à la censure grâce aux figures rhétoriques? », Romanic Review 109, 1-4, 2018, p. 5-30.

15. À plusieurs reprises, Félicité évoque sa peur de ne pas être crue; non pas parce que les événements qu'elle décrit n'ont pas eu lieu mais parce qu'ils sont trop incroyables au sens premier du mot, ils sont trop invraisemblables: «Tout ce que j'avais vu depuis le clocher, personne ne pourrait jamais le croire. À quoi bon témoigner quand le crime des crimes est impossible à comprendre? Par après, j'essayerai même de faire le ménage dans ma tête, me persuadant que rien n'était vrai. » (F. Lyamukuru \& N. Caprioli, L'ouragan a frappé Nyundo, op. cit., p. 175).

16. À ce propos, Piton note que cet attentat et l'accusation contre le FPR, a priori démentie par l'expertise de janvier 2012 du TGI de Paris, furent un puissant ressort de mobilisation contre la population tutsie (F. Piton, Le génocide des Tutsi du Rwanda, op. cit., p. 104).

17. Cf. note 10 de cet article.

18. Notons également que la symbolique du religieux fut importante lors de ce génocide: jusqu'alors, les lieux de culte étaient synonyme d'asile. Mais aussi, le père de Félicité officiait au petit séminaire de Nyundo. La rupture se marque donc doublement avec ce lieu sacré et la chapelle qui deviendra lieu de massacre.

19. F. Lyamukuru \& N Caprioli, L'ouragan a frappé Nyundo, op. cit., p. 102-103 \& 106. 
20. À ce propos, nous le soulignons, voir la réflexion de E. Danblon, Mandorla de Paul Celan, op. cit. 21. Pour une réflexion complète sur la notion rhétorique de vraisemblance, voir M. Kraus, "Nothing to Do with Truth? Eikos in Early Greek Rhetoric and Philosophy », dans L. Calboli Montefusco (éd.), Papers on Rhetoric, vol. VII, Rome, Herder, 2006. Voir aussi D. Hoffman, "Concerning Eikos: Social Expectation and Verisimilitude in Early Attic Rhetoric Author(s)", Rhetorica, vol. 26, 1, 2008, p. 1-29 ; S. di Piazza et F. Piazza (éd.), Verità verosimili. L'eikos nel pensiero grieco, Milan, Einaudi, 2013.

22. Ps.-Aristote, Rhétorique à Alexandre, texte introduit, éd. et trad. P. Chiron, Paris, Les Belles Lettres, CUF, 2002.

23. F. Goyet, Le regard rhétorique, Paris, Classiques Garnier, 2017, p. 57.

24. A ce propos, nous le disions, voir M. Dominicy, Poétique de l'évocation, op. cit. et E. Danblon, Mandorla de Paul Celan, op. cit.

25. E. Danblon, Mandorla de Paul Celan, op. cit.; et de la même auteure, La rhétorique comme exercice du regard. Les témoins, les prophètes ou comment se dit la clairvoyance? (cycle de conférence «les matins de la philosophie ", septembre-décembre 2019, Bruxelles, Belgique).

26. Fr. Goyet, Le regard rhétorique, op. cit, p. 34.

27. Par exemple, tandis que l'on peut penser savoir ce qu'est le saccage d'une ville, nous n'en aurons qu'une compréhension adéquate que lorsque nous aurons considéré les détails qui constituent un saccage de ville : les maisons brûlées, les enfants arrachés à leur mère, les femmes violées, etc.

28. Nous pensons par exemple à l'image en chiasme présente à la fin du discours entre « le verre de lait resté intacte» et les «bras et jambes coupés». Cet effet de chiasme participe de la compréhension de l'invraisemblable: a priori, un verre se brise plus facilement qu'un corps humain.

29. R. Webb, «Ekphrasis Ancient and Modern : The Invention of a Genre », Word \& Images vol. 15, 1, 1999, p. 7-18

30. Nous l'annoncions en début d'article, nous nous appuyons ici sur l'hypothèse développée par Ruth Webb dans « The Model Ekphraseis of Nikolaos the Sophist as Memory Images ", op. cit.

31. Manuel d'exercices rhétorique préparatoires. Nous avons conservé des versions de ces manuels faisant mention de l'ekphrasis, rédigés notamment par les auteurs suivants: Theon, Hermogène, Aphtonios ou encore Nikolaos.

32. R. Webb, « Ekphrasis Ancient and Modern : The Invention of a Genre », op. cit.

33. Pour une réflexion sur l'évidence voir C. Lévy \& L. Pernot (éd.), Dire l'évidence. Philosophie et rhétorique antique, Paris, L'Harmattan, 1997.

34. Nous reprenons ici la présentation de Quintilien proposée par Ruth Webb dans son article sur les ekphraseis de Nikolaos ("The Model Ekphraseis of Nikolaos the Sophist as Memory Images », op. cit.).

35. À ce sujet, Ruth Webb souligne les propos de Quintilien qui se plaint de la médiocrité des étudiants qui proposent des ekphraseis scolaires où la description, bien qu'excessive, ne laisse pas place à l'enargeia qui elle, peut naitre de la simple présence de l'un ou l'autre détail spécifique. Nous reprenons ici l'exemple de Quintilien du sac d'une ville que présente R. Webb dans «The Model Ekphraseis of Nikolaos the Sophist as Memory Images ", op. cit., p. 468.

36. À propos de l'importance de l'émotion dans le processus de persuasion, voir aussi M. Dominicy \& M. Frédéric (éd.), La mise en scène des valeurs. La rhétorique de l'éloge et du blâme, Lausanne, Delachaux et Niestlé, 2001.

37. Nous nous basons ici sur la présentation de l'auteur qui est proposée par Ruth Webb à partir de l'édition de 1913 du volume de Nikolaos. R. Webb, «The Model Ekphraseis of Nikolaos the Sophist as Memory Images ", op. cit., p. 468. 
38. Propos de Démosthène à propos du sac de la ville de Phokis par Philippe de Macédoine (19.65), présentés dans le manuel de Progymnasmata de Nikolaos (éd. J. Felten, Leipzig, 1913, p. 71).

39. Nous pensons ici à Cicéron qui rappelle que les tâches de l'orateur sont au nombre de trois : plaire, instruire et émouvoir (Cicéron, De l'orateur II, trad. E. Courbaud, Paris, Les Belles Lettres, 1928, p. 128-129).

40. Voir à ce propos A. Damasio, L'erreur de Descartes : la raison des émotions, Paris, Odile Jacob, 1995 ; mais aussi M. Dominicy \& M. Frédéric (éd.), La mise en scène des valeurs. La rhétorique de l'éloge et du blâme, op. cit.

41. M. Dominicy, Poétique de l'évocation, op. cit., p. 272.

42. E. Danblon, Mandorla de Paul Celan, op. cit.

43. Rappelons que l'ekphrasis fonctionne par synaesthesis: l'ensemble des perceptions sensorielles sont mobilisées.

44. M. Dominicy, Poétique de l'évocation, op. cit., p. 275.

45. En anglais dans l'original (dans R. Webb, «The Model Ekphraseis of Nikolaos the Sophist as Memory Images ", op. cit., p. 469, traduction libre) : "What is noticeable about this passage, and of great importance to the workings of ekphrasis in general, is that the action at which we are supposed to feel outrage (Philip's violence) is not described directly but through the visible traces it left. Yet in order for it to be effective, the audience must supply that information for themselves. The details described are therefore important above all as a sign of something else and we are left to infer the actions which preceded from the sketch of the aftermath alone. "

46. Libanios, Progymnasmata, éd. R. Foerster, Libanii Opera VIII, Leipzig, Teubner, 1915. Cité par Ruth Webb («The Model ... », op. cit., p. 474, traduction libre) : «In the case of the Trojan woman, the uncovered breast is said to show that her misfortunes have partially taken away her sense of shame (aidōs) and the narrator speculates that she is unaware of her partial nakedness a remark that portrays her as the unwitting object of the male gaze. ».

47. Ibid., p. 474.

48. Argument développé à partir d'un extrait du traité Rhetorica ad Herennium, III 20.33, mobilisé par Ruth Webb dans l'article cité précédemment : ibid., p. 473.

49. Notons quand même les distinctions suivantes : le contexte de production n'est pas ici un cadre scolaire et l'ekphrasis se manifeste ici sous une forme qui se rapproche plus de l'évocation telle que préconisé par Quintilien que de la description énumération telle que celle étudiée ici par Ruth Webb.

50. I. Assmann, La mémoire culturelle. Écriture, souvenir et imaginaire politique dans les civilisations antiques, Paris, Flammarion, 2010 [2002].

51. Ibid., p. 34.

52. Pour une réflexion sur les catégories de témoin oculaire, instrumentaire et historique, voir R. Dulong, «Qu'est-ce qu'un témoin historique ? », op. cit. et E. Danblon dans la présente livraison. Le témoin oculaire est celui qui est surpris par l'événement, qui ne peut savoir à l'avance qu'il sera témoin et qui subit la situation dont il est témoin. En quelque sorte, il s'autoinstitutionnalise témoin, il n'est mandaté par aucune institution pour remplir cette fonction. Le témoin instrumentaire désigne «l'agent qui exécute une tâche pour l'institution judiciaire, qui est mandaté pour cela et qui doit en rendre compte par un rapport écrit ». Le témoin historique est une sorte de requalification du témoin oculaire : il est le témoin oculaire, non-juridique, d'une situation (souvent catastrophique) qu'il a vécue et qu'il décrit. Plus spécifiquement, la catégorie de témoin historique "qualifie les auteurs de documents, publiés ou non, décrivant, pour les dénoncer, les catastrophes humaines » (R. Dulong, ibid.).

53. Aristote, Rhétorique, éd. et trad. P. Chiron, Paris, Garnier-Flammarion, 2007, p. 247.

54. R. Dulong, «Qu'est-ce qu'un témoin historique ?», op. cit. 


\section{AUTEUR}

\section{LUCIE DONCKIER DE DONCEEL}

Université libre de Bruxelles/Università degli Studi di Palermo 